\title{
ТРАЕКТОРИЯ РЕШЕНИЯ КОМБИНАТОРНЫХ И ВЕРОЯТНОСТНЫХ ЗАДАЧ ОТ ШКОЛЬНОЙ ПРОГРАММЫ ДО ПРИКЛАДНЫХ ДИСЦИПЛИН В ТЕХНИЧЕСКИХ ВУЗАХ
}

\section{THE TRAJECTORY OF SOLVING COMBINATORIAL AND PROBABILISTIC PROBLEMS FROM THE SCHOOL CURRICULUM TO APPLIED DISCIPLINES IN TECHNICAL UNIVERSITIES}

\section{Azizyan \\ E. Mironova \\ A. Sivirkina}

Summary: This article discusses some aspects of the study of combinatorics and probability theory in the context of continuity of school and University education. General problems of combinatorics are presented, and particular problems are considered in the framework of studying applied disciplines. The necessity of strengthening the probabilistic and statistical thinking of the student at all stages of teaching mathematics, for better assimilation of practical problems, is justified.

Keywords: combinatorics, probability theory, and reliability theory.
$\mathrm{B}$ результате освоения прикладных дисциплин математического цикла в политехническом вузе у обучающего формируются общепрофессиональные и профессиональные компетенции, которые можно охарактеризовать, как личностную способность решать определенный класс задач [1,14]. Например, в рамках изучения дисциплин математического цикла, способность к абстрактному мышлению, анализу, синтезу, применению системы фундаментальных знаний для идентификации, формулирования и решения технических задач.

В данной статье проследим траекторию изучения и решения комбинаторных и вероятностных задач от школьной программы до вузовского материала. Выбор данного раздела объясняется тем, что значимость комбинаторики не ограничивается математическим знанием, а предполагает у обучающегося развитие способности определять, рассматривать и учитывать все возможные варианты сочетания каких-либо признаков или событий, что служит предпосылкой логического рассуждения и формирования профессиональной компетентности.

Комбинаторика - раздел математики, в котором изучаются задачи выбора элементов из заданного множества и расположения их в группы по заданным правилам, в частности, задачи о подсчете числа комбинаций (выборок), получаемых из элементов заданного конеч-
Азизян Инара Артушовна

к.п.н., дочент, Рязанский институт филиал Московского политехнического университета inara_azizyan@mail.ru

Миронова Елена Ивановна

к.т.н., доцент, Рязанский институт филиал Московского политехнического университета el-konyaeva@yandex.ru

Сивиркина Анна Сергеевна к.n.н., доцент, Рязанский институт филиал Московского политехнического университета sivirkinaas@yandex.ru

Аннотация: В данной статье рассматриваются некоторые аспекты изучения комбинаторики и теории вероятностей в контексте преемственности школьного и вузовского образования. Представлены общие задачи комбинаторики, рассмотрены частные задачи в рамках изучения прикладных дисциплин. 0боснована необходимость усиления вероятностно-статистического мышления обучающегося, на всех этапах обучения математике, для лучшего усвоения задач практического характера.

Ключевые слова: комбинаторика, теория вероятностей, теория надежности.

ного множества [5].

Комбинаторика как раздел математики (алгебры) достаточно молодой, многие учителя не рассматривают и не воспринимают этот раздел как необходимый элемент школьного математического образования, который также требует детального изучения для глубокого знания теоретического материала, умения их использовать в контексте заданной проблемы, навыков по применению в задачах прикладного характера.

Целенаправленная работа по внедрению вопросов комбинаторики и вероятностно-статистической линии в школьный курс, с целью повышения математического образования, прослеживается в методике преподавания математики с 60-х годов в работах А.Н. Колмогорова, Б.В. Гнеденко и других. Спустя 20 лет теория вероятностей и комбинаторика рассматривались в школьной программе в качестве факультативного курса или раздела для дополнительного изучения. Далее были попытки изучения теории вероятностей и комбинаторики в основном разделе школьного курса, но полноценное внедрение можно считать с 2004-2008 гг. с выходом новых учебных пособий под авторством Макарычева Ю.Н., Ткачева М.В. и др.

В технических вузах в рабочую программу включена 
дисциплина «Математические основы теории надежности», которая является вычислительной базой для таких дисциплин как «Теория надежности и диагностики», «Инженерные расчеты конструкций» и т.д.

В рамках изучения данной дисциплины представим следующие частные задачи: найти вероятность безотказной работы устройства и определения относительной частоты годных приборов при испытании партий, с учетом правил сложения и умножения комбинаторики и схем с возвращением и без них; для определения числа стандартных и бракованных деталей и вероятности отказа одного элемента, хотя бы одного элемента, всех элементов системы и т. д.

Для решения данных задач предполагается наличие базовых понятий комбинаторики и теории вероятностей у студентов, которые они приобрели в школьном курсе математики. Однако, хотя и по учебной программе отводится определенное число часов, но в свете современных реалий, в рамках учебного процесса старшего звена, комбинаторика представляется в строго-формализованном виде и с учетом только тех заданий, которые так или иначе встречаются в контексте подготовки к единому государственному экзамену, что не способствует лучшему пониманию.

Сформулируем общие задачи комбинаторики следующим образом:

1. составить новое множество из заданного, состоящее в установлении определенного порядка следования элементов множества друг за другом, - составление перестановок;

2. составить подмножество из заданного множества, - составление сочетаний;

3. составить упорядоченное подмножество из заданного множества, - составление размещений.

Для решения данных задач необходимо сначала выстроить логическую базу для последующего математического решения.

Согласно опыту большинства школьных учителей самая большая проблема при решении комбинаторных задач заключается в формализации, переходе от вербальной формы к математической. В данном случае, это 1) опознание вида задачи (комбинаторная); 2) выбор комбинаторной схемы для решения; 3) применение формулы комбинаторики с конкретными числовыми данными [2].

Помимо непосредственного вычисления по формулам комбинаторики, в задачах прикладного характера используются ряд комбинаторных методов.

Рассмотрим несколько классов основных задач тео- рии надежности на основе комбинаторики, при решении которых используются:

- методы рекуррентных соотношений (решение поставленной задачи с определенным количеством предметов выражается через решение аналогичной задачи с меньшим числом предметов с помощью некоторого соотношения, которое называется рекуррентным (например, при расчетах условий для оптимального резервирования, для определения начальных моментов распределения числа нормально функционирующих исполнительных элементов и т.д.);

- метод включения и исключения (определение числа элементов объединения множеств, состоящий в поочередном сложении и вычитании, увеличении и уменьшении числа элементов), например, при расчете надежности невосстанавливаемых и восстанавливаемых резервированных систем (при дробном резервировании или системе типа “k из n", т. е. система состоит из n элементов, но для ее работоспособности нужно, чтобы не менее $\mathrm{k}$ из них были работоспособны);

- метод траекторий (геометрическая интерпретация (схемы, чертежи, графы), которая сводит задачу к подсчету числа путей (траекторий), обладающих определенным свойством (например, при определении связности графика для расчета надежности электрических систем при различном виде соединений).

Комбинаторные методы используются широко и в статистике, например, для оценки и контроля надежности технических устройств по результатам их испытаний.

В производстве приходится решать задачи: по определению надежности большой партии произведенных изделий; по выявлению количества деталей, не соответствующих конструкторской документации (КД); по распределению на поправимые и непоправимые.

Испытания технических устройств производятся с целью определения реального уровня их надежности. Испытаниям подвергается выборка из генеральной совокупности. При оценке и контроле основных критериев надежности используются инструменты комбинаторики: контроль числа дефектных изделий, контроль по наработке, при однократном, двукратном, последовательном наблюдении за результатами.

Контроль надежности имеет своей целью проверить гипотезу о том, что надежность рассматриваемого объекта (системы, партии) не ниже установленного уровня.

Так как контроль надежности производится на основе анализа элементов выборки, то при принятии решений возможны два вида ошибок: первого рода - когда 
хорошая партия не принимается из-за несоответствия КД; второго рода - когда плохая партия готовой продукции принимается. Вероятность ошибки первого рода называется риском поставщика (a), а второго рода - риском заказчика ( $\beta)$.

Рассмотрим метод однократной выборки: из контролируемой партии готовой продукции большого объема (N) берется партия меньшего объема (n), сформированная случайным образом. Исходя из исходных данных, устанавливаются оценочные нормативы ( $A_{0}$ и $\left.A_{1}\right)$. Если выборочное значение контролируемого параметра меньше или равно $\mathrm{A}_{0}$, то партия признается надежной; если больше или равно $A_{1}$, то партия бракуется. При решении задач в качестве исходных данных необходимо учитывать ряд условий: ограничения по принятию партии, объем испытаний и т. д. Пусть требуется оценить надежность малой контрольной партии изделий из 50 экземпляров. В качестве опорных точек можно выбрать вероятности принятия партии: партия хорошая, если в ней содержится не более 10 процентов дефектных изделий, и плохая - при содержании 20 процентов дефектных изделий. Риск заказчика (частной компании) и риск поставщика (завода) оценим по 10 процентов. Необходимо определить оптимальные значения приемочного и браковочного числа.

При решении практической задачи достаточно случайным образом выбрать 20 изделий из 50. Такое количество изделий дает возможность провести расчеты на основе гипергеометрического распределения.

Десять процентов от нашей партии $\mathrm{D}_{0}$ составляет 5 изделий, а при $20 \%$ дефектных изделий $\mathrm{D}_{1}-10$ изделий.

Определение приемочного числа дефектных изделий проводятся с помощью формул комбинаторики (расчет числа сочетаний различных комбинаций). Суммирование вероятностей гипергеометрического распределения производится до тех пор, пока накопленная вероятность: риск поставщика не приблизится к 90 процентам, а браковочное число (риск заказчика) к 10 процентам. В интересах заказчика уменьшение браковочного числа готовой продукции, в интересах завода - увеличение числа изделий, не соответствующих конструкторской документации.

Рассматривая серию прикладных задач, мы приходим к выводу, что в настоящее время, комбинаторика и теория вероятностей заложены в теоретическую и практическую основу естественных и технических дисциплин, организацию производства, экономику, планирование и прогнозирование.

Чтобы обеспечить качественную преемственность при изучении представленных вопросов, необходимо, начиная с начального этапа школьного образования уделять должное внимание формированию вероятностно-статистического мышления, умению оперирования методами перебора и выбора комбинаторики.

\section{ЛИТЕРАТУРА}

1. Азизян, И.А. Значимость языковой составляющей в процессе формирования общепрофессиональных и профессиональных компетенций студентов политехнического вуза//Вопросы педагогики, 2018, №6, с. 14-16.

2. Драгныш, Н.В. Визуализация комбинаторных задач теории вероятностей // Молодой ученый. — 2016. — №15. — C. 129-133.

3. Гмурман, В.Е. Руководство к решению задач по теории вероятностей и математической статистике.- М., 1998, 400 c.

4. Острейковский, В.А. Теория надежности. $-\mathrm{M}, 2003,463$ c.

5. Письменный, Д.Т. Конспект лекций по теории вероятностей, математической статистике и случайным процессам. - М., 2007, 288 c.

6. Половко, А.М., Гуров С.В. Основы теории надежности. Практикум. - СПб, 2006, 560 с.

7. Ушаков, И.А. Курс теории надежности систем. - М., 2008, 239 С.

8. Фадеева, Л.Н., Жуков, Ю.В., Лебедев, А.В. Математика для экономистов: Теория вероятностей и математическая статистика. Задачи и упражнения. - М., 2006, $336 c$

(с) Азизян Инара Артушовна (inara_azizyan@mail.ru), Миронова Елена Ивановна (el-konyaeva@yandex.ru), Сивиркина Анна Сергеевна (sivirkinaas@yandex.ru). 